\title{
ERRATUM
}

Toshihiko Takaya $\cdot$ Hidenori Kawamura

Yoshihiro Minagawa • Masahito Yamamoto

Azuma Ohuchi

\section{PID landing orbit motion controller for an indoor blimp robot}

\section{Artif Life Robotics (2006) 10:177-184 \\ DOI 10.1007/s10015-006-0385-9}

The authors' affiliations appeared incorrectedly in the article cited above.

The correct authors' affiliations are as follows.

T. Takaya $(\square)$

Ricoh Software, Nishi 4 chome, Kita 7 jyo, Kita-ku Sapporo,

Hokkaido 060-0807, Japan

Tel. +81-11-716-7660; Fax +81-11-716-7665

e-mail: ttakaya@mountain.ocn.ne.jp

T. Takaya

Graduate School of Engineering, Hokkaido University, Hokkaido,

Japan

H. Kawamura $\cdot$ Y. Minagawa $\cdot$ M. Yamamoto $\cdot$ A. Ohuchi

Graduate School of Information Science and Technology, Hokkaido

University, Hokkaido, Japan 